\title{
Electromagnetic Radiation Generated by Acoustic Excitation of Rock Samples
}

\author{
Lyudmila V. YAVOROVICH ${ }^{1}$, Anatolii A. BESPALKO ${ }^{1}$, \\ Pavel I. FEDOTOV ${ }^{1}$, and Rina B. BAKSHT ${ }^{2}$ \\ ${ }^{1}$ Institute of Non-Destructive Testing, National Research Tomsk Polytechnic \\ University, Tomsk, Russia; e-mail: pif@tpu.ru (corresponding author) \\ ${ }^{2}$ Institute of High Current Electronics, \\ Siberian Branch of the Russian Academy of Sciences, Tomsk, Russia
}

\begin{abstract}
The paper presents an experiment on acoustic excitation of electromagnetic radiation (EMR) signals in skarn, sandstone, and magnetite ore samples. For the skarn and sandstone samples, the EMR signal amplitude was observed to decrease with increasing ultimate strength. Supposedly, this effect can be explained by assuming that EMR is generated when an acoustic wave propagates through an electrical double layer. The presence of piezoelectric inclusions (e.g., quartz) in the magnetite ore enhances the analog EMR signal and its spectral components.
\end{abstract}

Key words: rocks, electromagnetic radiation (EMR), skarn, magnetite ore, sandstone, artificial acoustic excitation.

\section{INTRODUCTION}

Electromagnetic radiation (EMR) arising as a result of mechanical loading of rocks has been studied for many years (Warwick et al. 1982, Scholz 1968, Gol'd et al. 1975, Koktavy 2009, Lacidogna et al. 2011, Baddari et al. 2015). The main motive in these studies is the prospects for using this type of EMR to forecast geodynamic events, such as earthquakes (Baddari et al.

Ownership: Institute of Geophysics, Polish Academy of Sciences

(C) 2016 Yavorovich et al. This is an open access article distributed under the Creative CommonsAttribution-NonCommercial-NoDerivs license

http://creativecommons.org/licenses/by-nc-nd/3.0/. 
1999, Fidani 2011), rock bursts, and sudden roof failure in mines and quarries (see, e.g., Frid and Vozoff 2005, Bespalko et al. 2010).

It is well known that the fracture of materials is accompanied by generation of EMR. Currently, the mechanism of the EMR generation during the loading and cracking of materials is not understood in detail. The EMR emitted during the nucleation and traveling of a local mechanical disturbance in deformed rock can be accounted for by the formation of charged dislocations, electrokinetic phenomena, dislocation and discharge processes, and the like (Baddari et al. 1999). Some experimental facts related to the formation of cracks can be well explained by the surface oscillation model (Frid et al. 2003, Lacidogna et al. 2011). According to this model, EMR is generated by oscillating dipoles created by ions moving collectively as a surface wave over both faces of a crack formed in a material during its fracture.

The EMR in rocks is known to be associated with both the formation of microcracks and the propagation of acoustic waves through the material (Khatiashvili and Perel'man 1989, Lacidogna et al. 2013). Thus, a dipole exciting EMR appears due to the existence of electrical double layers in rocks (Perel'man and Khatiashvili 1983). Electrical double layers (EDLs) are formed at the boundaries of mineral grains and mineralized fluid inclusions and on the banks of microcracks and interstices. The EMR induced by an acoustic wave propagating through a double layer was first observed in a model experiment (Perel'man and Khatiashvili 1983). A pile of parallel thin sheets of glass, each aluminized on one side, was immersed in distilled water and driven by a piezoelectric transducer at a frequency ranging between 20 and $150 \mathrm{kHz}$. The recorded EMR signals had amplitude-frequency characteristics similar to those of the respective acoustic signals.

In the last few years, a number of laboratory experiments have been performed to investigate the EMR generated on compression and fracture of rock samples (Lacidogna et al. 2011, Koktavy 2009, Sobolev et al. 2010, Kobayashi et al. 2014). The reverse seismoelectric effect, i.e., generation of elastic vibrations by a rock in an AC electric field, is also essential to the understanding of the EMR physics (Ponomarev et al. 2002, Sobotka 2004, $2009 \mathrm{a}, \mathrm{b})$. The investigations were aimed both at verifying some EMR generation models (Frid et al. 2003, Koktavy et al. 2004, Koktavy 2009) and at finding relationships between the rock properties and the associated electromagnetic phenomena (Sobolev et al. 2010, Wan et al. 2008, Kobayashi et al. 2014). Recently, neutron bursts have been detected to occur on fracture of quasi-brittle materials such as rocks. This phenomenon is discussed elsewhere (Carpinteri et al. 2010, 2012, 2013; Borla et al. 2015). The EMR generated by acoustic excitation was investigated either with ionic crystals $(\mathrm{LiF}$, $\mathrm{NaCl}, \mathrm{KCl}$; Khatiashvili and Perel'man 1989) or with crystalline materials $\left(\mathrm{CaCO}_{3}\right.$; Khatiashvili and Perel'man 1989, quartz; Bespalko et al. 2005). 
However, no study was performed for the EMR generated by the acoustic excitation of rock samples with different content of the crystalline and amorphous inclusions.

In our opinion, to obtain a more comprehensive knowledge of the phenomenon of EMR generation, it would be useful to investigate how the properties of rocks affect the electromagnetic phenomena that occur on acoustic excitation of EMR. In addition, these investigations would perhaps clarify whether the EDL model can be used to explain adequately the EMR generation in rocks. In the experiment presented below, EMR was excited by passing an acoustic signal generated by a piezoelectric radiator through a rock sample. Note that an artificial source of acoustic waves was previously used to examine acoustic emission in rock samples (Sedlak et al. 2008, Cs'efalvay and Sedl'ak 2012). The aim of our study was to demonstrate the features of the EMR generation on acoustic excitation of rock samples of varied properties.

\section{EXPERIMENTAL SETUP}

This section presents the main characteristics of the artificial acoustic excitation source and the technique of EMR recording. Figure 1 shows a block diagram of the experimental setup.
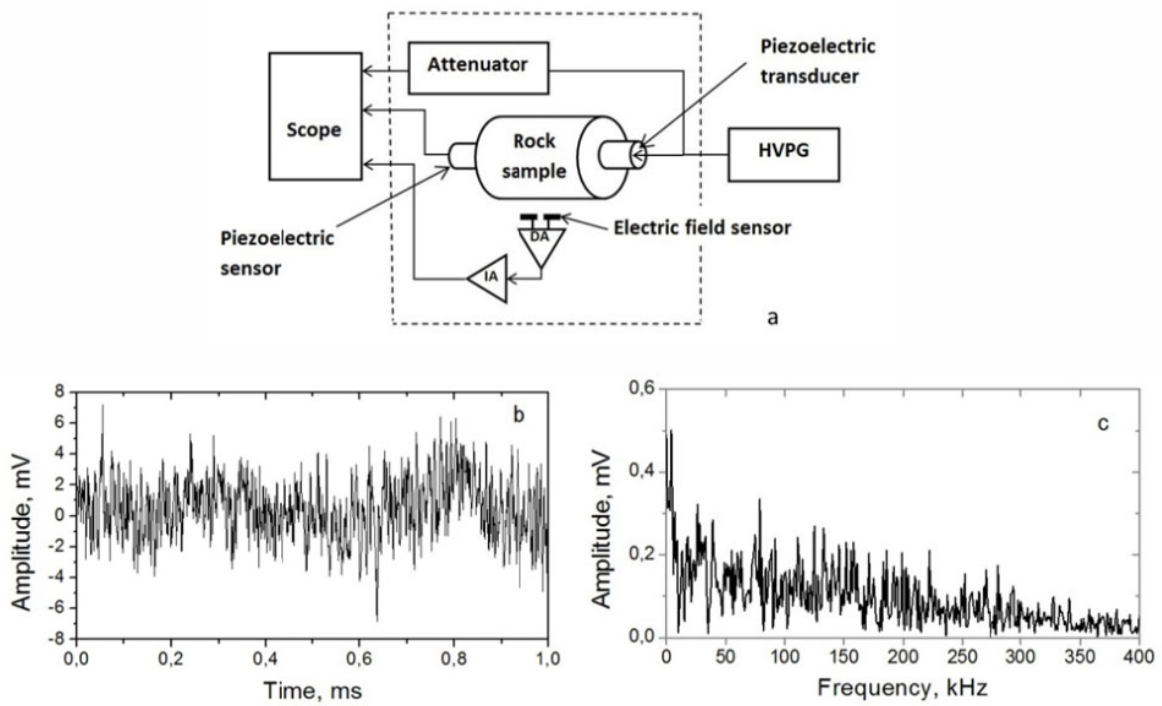

Fig. 1: (a) Block diagram of the experimental setup: HVPG - high-voltage pulse generator whose pulses are fed to the piezoelectric transducer, DA and IA - differential and intermediate amplifiers that provide recording of EMR; (b) analog noise signal; and (c) amplitude-frequency spectrum of the noise signal recorded with no sample on the X-Y table. 


\subsection{Acoustic excitation}

The source of the acoustic signal was a piezoelectric transducer made on the base of PZT-19 ceramics (http://www.elpapiezo). The rectangular pulses fed to the transducer were produced by a high-voltage pulse generator (HVPG). The HVPG pulse duration and voltage can be varied in the range of $10^{-6}$ $10^{-4} \mathrm{~s}$ and in the range of $100-800 \mathrm{~V}$, respectively. A broadband $(1-100 \mathrm{kHz})$ piezoelectric sensor, also made on the base of PZT-19 ceramics, was used for recording the acoustic signal transmitted through the sample. The duration of the rectangular acoustic signal incident on the sample is $5 \mu$ s. While passing through the sample, the signal is multiply reflected from its end faces. As a result, the signal converted into package of acoustic waves with dominant frequencies of 29 and $67 \mathrm{kHz}$. The signal generated by the piezoelectric sensor was recorded by a Tektronix TDS2024 oscilloscope. In the experiment, the HVPG pulse duration was $5 \mu$ s and the exciting voltage of the piezoelectric transducer was $800 \mathrm{~V}$.

\subsection{EMR signal recording}

The test rock sample, shaped like a cylinder, was clamped. The acoustic signal produced by the piezoelectric transducer, while passing through the sample, generated EMR. The EMR signal was detected using an electric field sensor (EFS) located on an X-Y table $2 \mathrm{~mm}$ away from the sample. The use of an X-Y table enabled high precision positioning of the EFS near the sample. The EFS consisted of two copper plates whose length and width were both $1 \mathrm{~cm}$ and thickness was $0.3 \mathrm{~cm}$. The plates were bent so that the spacing between the sample side surface and the plate plane was $2 \mathrm{~mm}$. Each plate was connected to its own input of a differential amplifier (DA) with input resistance of more than $30 \mathrm{M} \Omega$. DA is a two-input electronic amplifier whose output signal is the difference between the input voltages. In the DA, noise was suppressed and the useful signal arrived at an intermediate amplifier (IA) with a gain equal to 100 . Figures $1 \mathrm{~b}$ and $1 \mathrm{c}$ present the noise signal and its amplitude-frequency spectrum recorded with no sample on the X-Y table. It can be seen that the amplitude of the EMR noise component was about $4 \mathrm{mV}$ and that of the spectrum was $0.3 \mathrm{mV}$. Having passed through the IA, the signal arrived at the input of a Tektronix TDS2024 oscilloscope, whose input resistance $(1 \mathrm{M} \Omega)$ is significantly greater than the output resistance of the IA $(2 \mathrm{\kappa} \Omega)$. Noteworthy is the high degree of reproducibility of the EMR signal generated in the acoustically excited samples. For a series of ten sequential shots with the same sample, the statistical average deviation was not over $5 \%$. 


\section{EXPERIMENTAL RESULTS}

In our experiment, we examined the behavior of the EMR amplitude and frequency characteristics (EMR Fourier spectrum) for rock samples with different magnetite contents. We used samples of metamorphic rock and sedimentary rock (Tashtagol, Western Siberia). The samples of metamorphic rock were represented by skarn and magnetite ore and the samples of sedimentary rock by sandstone. The metamorphic samples were cut from a core and shaped into cylinders of diameter $(42 \pm 1) \mathrm{mm}$ and height $(80 \pm 2) \mathrm{mm}$. The sandstone samples were also cut from a core and shaped into cylinders of diameter $(30 \pm 1) \mathrm{mm}$ and height $(42 \pm 2) \mathrm{mm}$. The end faces of the cylinders were polished flat and parallel to within $(0.5 \pm 0.1) \mathrm{deg}$; the axis of a sample and its ends were at an angle of $(90 \pm 1) \mathrm{deg}$. Prior to being tested, all samples were subjected to petrographic analysis. Based on the analysis data, we divided the samples into three groups: a group of skarn (samples S1, S2, and S3), a group of magnetite ore (samples M1, M2, M3, and M4), and a group of sandstone (samples Sa1, Sa2, Sa3, and Sa4). For the metamorphic samples, x-ray analysis was also performed. We determined the mineral contents and some lithological parameters of our samples. The Protodyakonov rock hardness (Matti 1999) for our metamorphic samples was equal to 14. The skarn sample compositions were 50\% epidote, $30 \%$ garnet, and $20 \%$ chlorite (S1); $62 \%$ epidote, $28 \%$ garnet, and 10\% chlorite (S2); and 70\% epidote, $20 \%$ garnet, and $20 \%$ chlorite (S3). The magnetite ore sample compositions were $11.7 \%$ magnetite, $12 \%$ quartz, $30 \%$ garnet, $20 \%$ chlorite, and $26 \%$ calcite (M1); $11.7 \%$ magnetite, $3 \%$ quartz, $55 \%$ epidote, and $30 \%$ calcite (M2); $18.9 \%$ magnetite, $31 \%$ epidote, $26 \%$ amphibole, and $25 \%$ calcite (M3), and $21.05 \%$ magnetite, $15 \%$ garnet, $38 \%$ epidote, and $25 \%$ calcite (M4). The quartz content in samples S1, S2, S3, M3, and M4 was below the sensitivity level of the detecting equipment. Table 1 presents in detail the mineral composition of the sandstone samples.

Table 1

Mineral composition and porosity of the sandstone samples

\begin{tabular}{|c|c|c|c|c|c|c|c|c|}
\hline & & Po- & \multicolumn{3}{|c|}{ Minerals [\%] } & \multicolumn{2}{c|}{ Fragments of rocks [\%] } \\
\cline { 4 - 9 } Sample & Clast size & $\begin{array}{c}\text { rosit } \\
\text { [\%] }\end{array}$ & $\begin{array}{c}\text { Quartz } \\
\text { sand }\end{array}$ & $\begin{array}{c}\text { Feld- } \\
\text { spars }\end{array}$ & Pyrite & $\begin{array}{c}\text { Sedimentary } \\
\text { and } \\
\text { metamorphic }\end{array}$ & Effusive & $\begin{array}{c}\text { Grani- } \\
\text { toid }\end{array}$ \\
\hline Sa1 & medium & 16.8 & 60 & 11 & 2 & 13 & 9 & 5 \\
Sa2 & medium & 16.9 & 64 & 12 & 1 & 15 & 4 & 4 \\
Sa3 & coarse & 12.7 & 62 & 9 & 2 & 16 & 5 & 6 \\
Sa4 & coarse & 13.1 & 62 & 12 & - & 16 & 5 & 5 \\
\hline
\end{tabular}



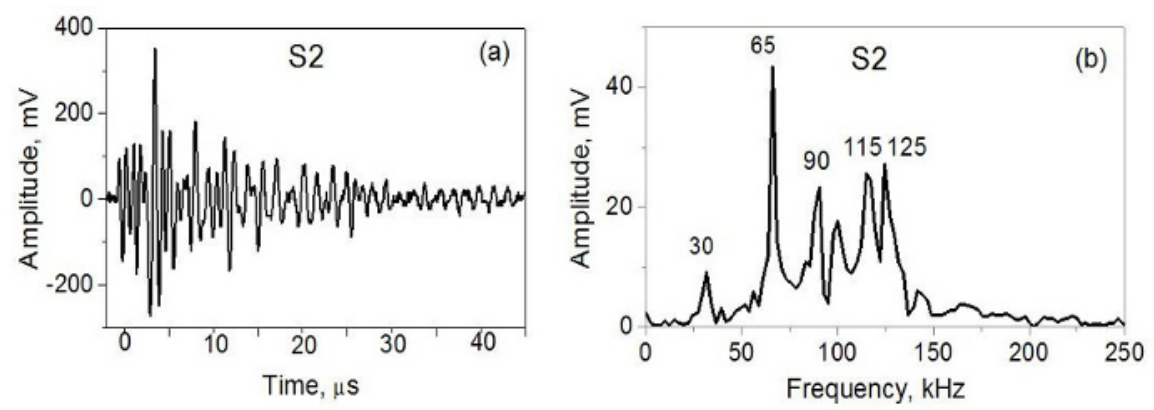

Fig. 2. EMR waveform for acoustically excited sample S2 (a) and its Fourier spectrum (b).

Figure 2 shows an EMR signal (a) generated on excitation of skarn sample S2. The respective Fourier spectrum is shown in Fig. 2b. As can be seen in Fig. 2b, the peak voltage amplitudes in the spectrum occur in the frequency bands of about $30,65,90,100,115$, and $125 \mathrm{kHz}$. In the same frequency bands, peak voltage amplitudes are observed for skarn samples S2 and S3.

The Fourier spectra for the skarn samples are characterized by a peak at a frequency of $65 \mathrm{kHz}$. The peaks at 30 and $65 \mathrm{kHz}$ approximately correspond to the dominant frequencies detected in the acoustic signal. A similar EMR spectrum was obtained by the Frid et al. (2003) who recorded the EMR signal generated during the fracture of granite on compression. Using the peak voltage amplitudes that occurred in these frequency bands, we have plotted the peak amplitude as a function of frequency for the skarn samples (Fig. 3).

The EMR signals generated on excitation of sandstone samples are similar to the EMR signals of the skarn samples: the Fourier spectra for the sand-

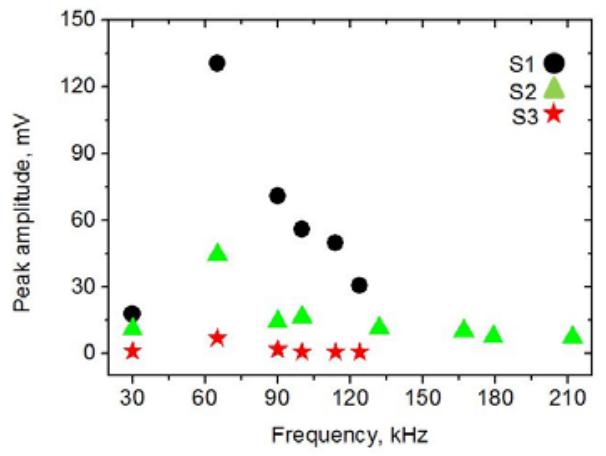

Fig. 3. Peak voltage amplitude versus EMR spectrum component frequency for samples S1, S2, and S3. 

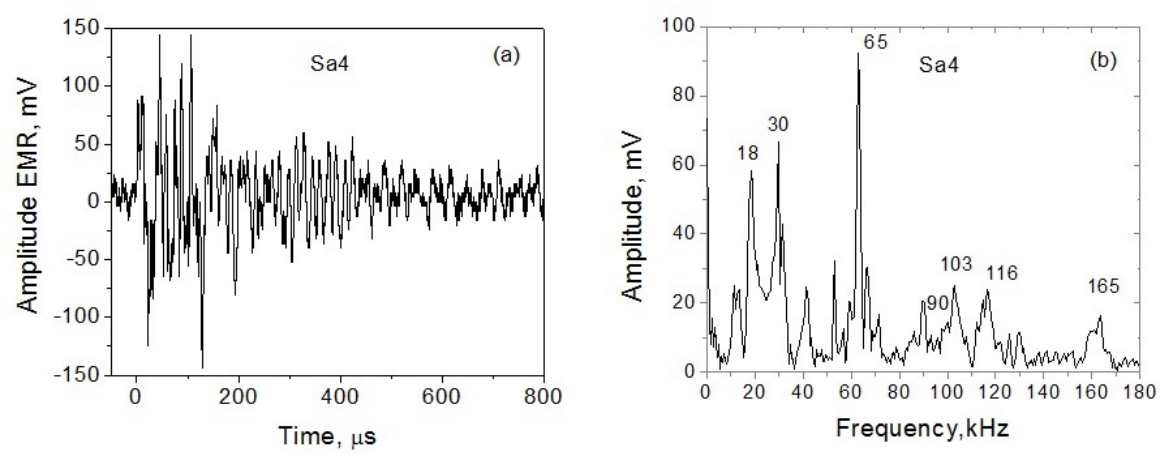

Fig. 4. EMR waveform for acoustically excited sample S2 (a) and its Fourier spectrum (b).

stone samples also have a peak at a frequency of $65 \mathrm{kHz}$. Figure 4 shows an EMR signal (a) generated on excitation of sandstone Sa2. The respective Fourier spectrum is shown in Fig. 4b. Figure 5 shows the peak voltage amplitude versus EMR spectrum component frequency for samples $\mathrm{Sa} 1, \mathrm{Sa}$, $\mathrm{Sa} \mathrm{3}$, and $\mathrm{Sa} 4$. The peaks at 30 and $65 \mathrm{kHz}$ also correspond to the dominant frequency detected in the acoustic signal that we observed earlier for the skarn samples.

The samples of magnetite ore excited with an acoustic signal behaved quite differently than the skarn and sandstone samples. That is why we give the EMR signal waveforms for all four test samples. Figure $6 \mathrm{a}, \mathrm{c}$, e, and $\mathrm{g}$ presents the electromagnetic signals generated on excitation of magnetite ore samples M2, M3, and M4. The Fourier spectra corresponding to these signals are shown in Fig. 6b, d, f, and $h$.

It can be seen that the Fourier spectra obtained for magnetite ore (see Fig. $6 \mathrm{~b}, \mathrm{~d}$, and $\mathrm{h}$ ) have no pronounced peak. Furthermore, the frequency at

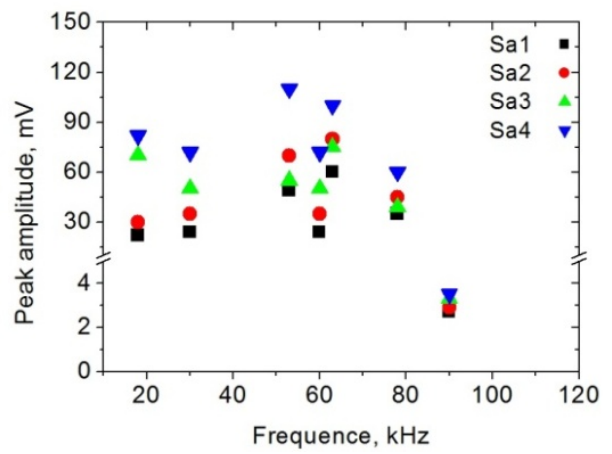

Fig. 5. Peak voltage amplitude versus EMR spectrum component frequency for samples $\mathrm{Sa} 1, \mathrm{Sa} 2, \mathrm{Sa} 3$, and $\mathrm{Sa} 4$. 

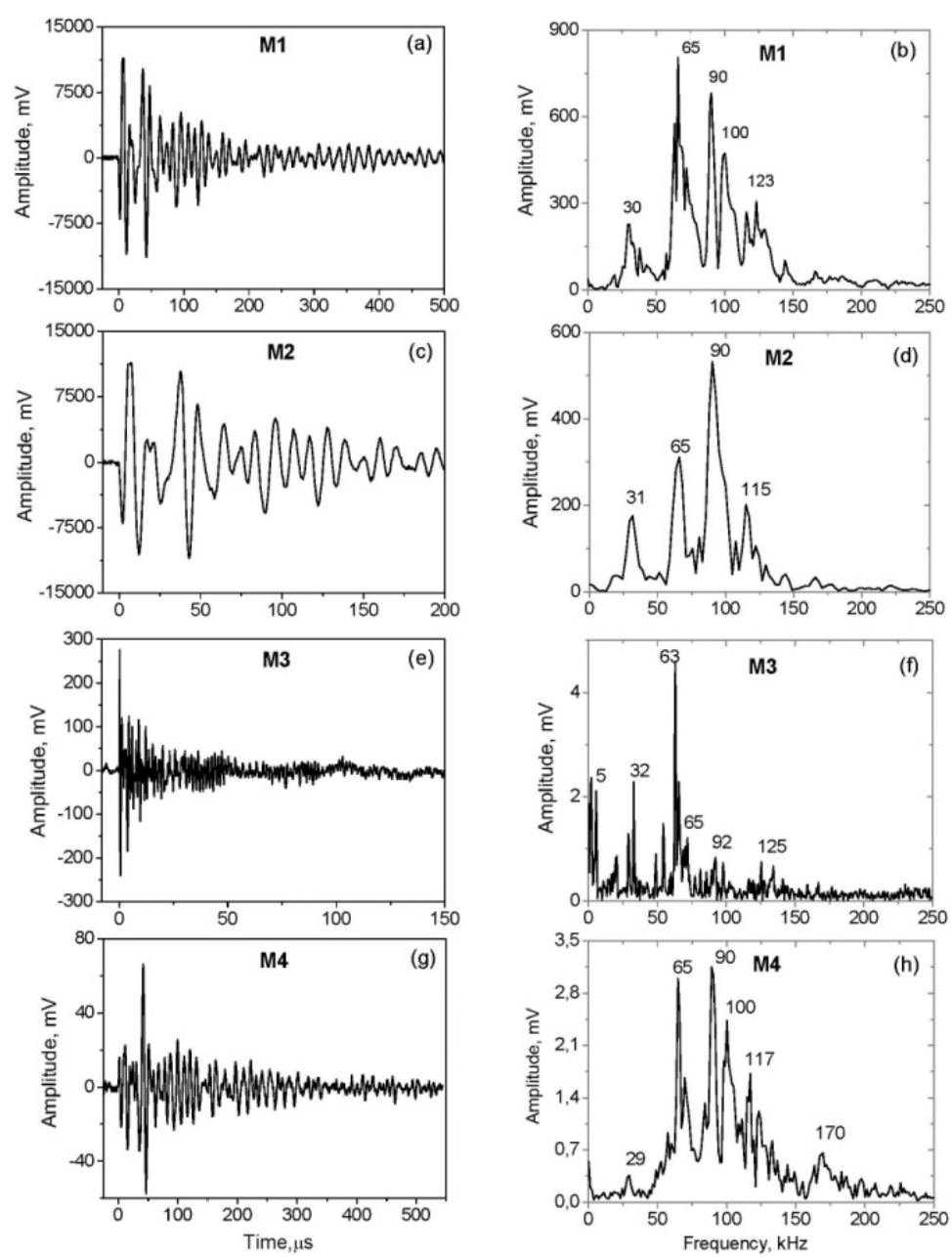

Fig. 6. EMR waveforms for acoustically excited samples M1 through M4 (a-g) and the respective Fourier spectra (b-h).

which the most intense EMR was detected varied from sample to sample. Thus, for samples M1 and M2, the highest voltage peak in the Fourier spectrum corresponds to 65 and $90 \mathrm{kHz}$, respectively (Fig. 7).

The Fourier spectrum voltage amplitudes at $65 \mathrm{kHz}$ and $90 \mathrm{kHz}$ are given for all samples in Table 2 together with the sample electric resistivity $\eta$ and ultimate strength Pult. The measurements of $\eta$ and Pult were performed after the experiment on acoustic excitation of EMR had been completed. The resistivity $\eta$ was measured by the technique described by Kani (1985). To determine the ultimate strength Pult, the samples were subjected to uniaxial 


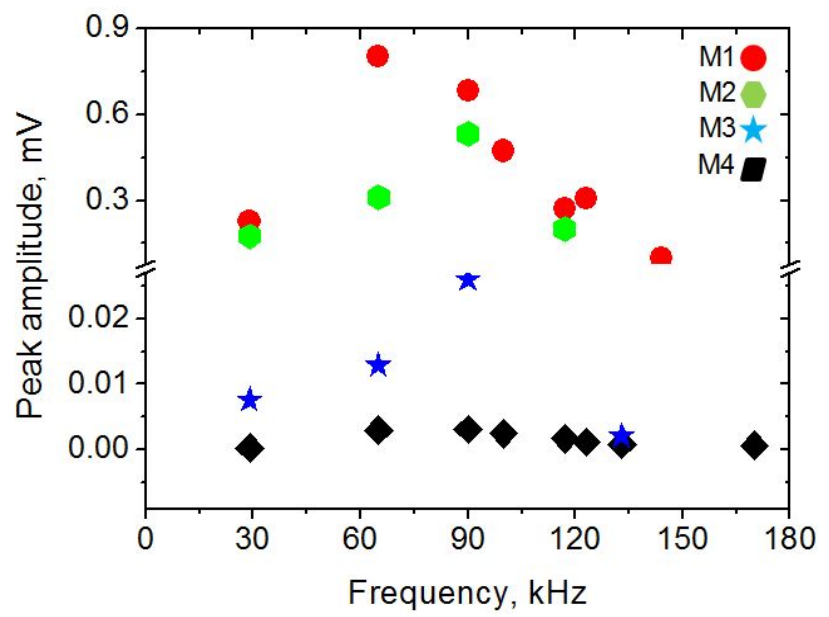

Fig. 7. Peak voltage amplitude versus EMR spectrum component frequency for samples M1, M2, M3, and M4.

Table 2

Fourier spectrum voltage amplitudes

\begin{tabular}{|c|c|c|c|c|c|c|}
\hline \multirow{2}{*}{ Sample } & \multirow{2}{*}{$\begin{array}{c}\text { Ultimate } \\
\text { strength } \\
P_{\text {ult }} \\
{[\mathrm{kN}]}\end{array}$} & \multirow{2}{*}{$\begin{array}{c}\text { Resistivity } \\
\eta \\
{[\Omega \cdot \mathrm{m}]}\end{array}$} & \multirow{2}{*}{$\begin{array}{c}\text { Density } \\
\rho \\
{\left[\mathrm{g} / \mathrm{cm}^{3}\right]}\end{array}$} & \multirow{2}{*}{$\begin{array}{c}\text { Magnetite } \\
\text { content } \\
{[\%]}\end{array}$} & \multicolumn{2}{|c|}{$\begin{array}{c}\text { EMR amplitude } \\
{[\mathrm{mV}]}\end{array}$} \\
\hline & & & & & $65 \mathrm{kHz}$ & $90 \mathrm{kHz}$ \\
\hline \multicolumn{7}{|l|}{ Skarn } \\
\hline S1 & 188 & $4.5 \times 10^{3}$ & 2.9 & 0 & 135 & 70 \\
\hline $\mathrm{S} 2$ & 234 & $5.1 \times 10^{3}$ & 3.1 & 0 & 40 & 14.5 \\
\hline S3 & 320 & $4.8 \times 10^{3}$ & 2.8 & 0 & 8 & 2 \\
\hline \multicolumn{7}{|c|}{ Magnetite ore } \\
\hline M1 & 234 & 16 & 3.4 & $11.7 \pm 1$ & 800 & 680 \\
\hline M2 & 193 & 17 & 3.4 & $11.7 \pm 1$ & 350 & 530 \\
\hline M3 & 317 & 19 & 3.7 & $18.9 \pm 1$ & 12 & 0.8 \\
\hline M4 & 258 & 21 & 3.8 & $21.05 \pm 1$ & 3 & 3.4 \\
\hline \multicolumn{7}{|l|}{ Sandstone } \\
\hline Sa1 & 25 & $7 \times 10^{5}$ & 2.19 & 0 & 100 & 3.5 \\
\hline $\mathrm{Sa} 2$ & 28 & $6.5 \times 10^{5}$ & 2.16 & 0 & 80 & 3.3 \\
\hline $\mathrm{Sa} 3$ & 35 & $9 \times 10^{4}$ & 1.97 & 0 & 75 & 2.9 \\
\hline $\mathrm{Sa} 4$ & 40 & $1.5 \times 10^{4}$ & 1.99 & 0 & 65 & 2.7 \\
\hline
\end{tabular}


compression on an SP-500 press. Table 2 also gives the material density $\rho$ and the magnetite content for each sample.

The magnetite content in the test samples, $V_{\mathrm{m}}$, was estimated by the formula

$$
V_{m}=V_{s}\left[1-\left(\rho_{s}-\rho_{m}\right) /\left(\rho_{s}-\rho_{h}\right)\right]
$$

where $V_{\mathrm{s}}$ is the sample volume; $\rho_{s}$ is the mean density of the sample; $\rho_{m}$ is the density of magnetite ore, $\rho_{m} \approx 5 \mathrm{~g} / \mathrm{cm}^{3} ; \rho_{h}$ is the density of the host rock, $\rho_{h} \approx 3 \mathrm{~g} / \mathrm{cm}^{3}$ (in our case). The volume $V_{\mathrm{s}}$ was the same for all magnetite samples and equaled $402 \mathrm{~cm}^{3}$.

As follows from the data presented in Table 2, the resistivity correlates well with the percentage of magnetite ore. In addition, it can clearly be seen that for the skarn and sandstone samples, the maximum amplitude of the Fourier spectrum is inversely proportional to ultimate strength. At the same time, analysis of the data for the magnetite ore samples (see Table 2 and Fig. 6) has revealed no pronounced dependence of the amplitude of the EMR spectrum components on the strength.

\section{DISCUSSION}

Skarn. One of the main findings of this experiment was that for the skarn samples the amplitude of the main component the Fourier spectrum $(65 \mathrm{kHz})$ of the EMR signal decreased almost tenfold as the ultimate strength $P_{\text {ult }}$ increased from 188 to $320 \mathrm{kN}$ (see Table 2). It is well known that skarns bear pores filled with saline fluid as well as microcracks of varied orientation and dimensions (Mavko et al. 2009). These violations of the homogeneity of the skarn samples resulted in the formation of electrical double layers (EDLs). Compression and expansion of the rock that contained an EDL converted the exciting acoustic pulse into an EMR signal.

A flat EDL can be considered as a system of three parallel-connected capacitors corresponding to the space charge region in the dielectric, the Helmholtz layer on the surface, and the Gouy layer in the electrolyte (Khatiashvili and Perel'man 1989). According to Khatiashvili and Perel'man $(1983,1989)$, the effective thickness of the first and third capacitors is determined by the Debye radius $r_{i}=\left(\varepsilon k T / 4 p e^{2} z^{2} n_{i}\right)^{0.5}$, where $\varepsilon$ is the real part of permittivity, $z$ is the ion valence, and $n_{i}$ is the redundant charge density. The electrostatic EDL energy per unit area can be described as

$$
E=\sum E_{i}=\left(\frac{\pi k T}{e^{2} z^{2}}\right)^{0.5} \times \sum \frac{1}{\sqrt{\varepsilon n_{i}}}
$$

If such a system is exposed to a pressure $p(t)$, its energy increases with decreasing product $\varepsilon n_{i}$ in the extension stage and decreases in the compres- 
sion stage. In this case, some portion of the excess electrostatic energy should be radiated and the other, given high conductivity, can be released in the medium as Joule heat. Khatiashvili and Perel'man (1989) suggest to neglect the effect of pressure on the characteristics of the Helmholtz layer. Then the change in energy of the double layer "capacitors" can be described as

$$
\Delta E(t)=-0.5 p(t)\left\{\frac{2 \varepsilon_{1}-1}{\varepsilon_{1} Y} E_{1,0}+\frac{2 \varepsilon_{3}-1}{\varepsilon_{3}} \alpha_{v} E_{3,0}\right\}
$$

where $E_{1,0}$ is the initial energy associated with the space charge, $E_{3,0}$ is the initial energy associated with the Gouy-Chapman layer of the electrolyte, $Y$ is Young's modulus, and $\alpha_{V}$ is the volumetric thermal expansion coefficient. The acoustic pressure is defined as $p(t)=\omega A$, where $\omega$ and $A$ are the acoustic field frequency and magnitude. Hence, the amplitude $I_{\mathrm{EMR}}$ of the EMR signal generated by a set of synchronized EDLs in an acoustic field of frequency $\omega$ can be estimated as (Khatiashvili and Perel'man 1989):

$$
I_{E M R}=\frac{\omega \Delta E S}{\pi} \approx \frac{v \rho \omega^{2} S A(2 \varepsilon-1)}{\varepsilon_{1} \pi} \times \frac{E_{1,0}}{Y}+\frac{v \rho \omega^{2} S A(2 \varepsilon-1)}{\varepsilon_{1} \pi} \alpha_{v} E_{3,0^{\prime}}
$$

where $S$ is the total area of the EDLs, $v_{\mathrm{s}}$ is the sound velocity, and c is the mean density of the rock. The second term in (2) is related to the diffuse space charge in the Gouy-Chapman layer. The first term in (2) describes the energy change in a thin capillary crack where the Gouy-Chapman layers adhering to the opposite walls of the crack overlap. This term indicates that the EMR amplitude $I_{\text {EMR }}$ decreases with increasing Young's modulus. It is well known that Young's modulus $Y$ is directly proportional to ultimate strength $P_{\text {ult }}$ (Mavko et al. 2009). Thus, the experimental relation that we have found for skarns, $I_{\mathrm{EMR}} \propto \omega^{2}$ (see Table 2), can be reasonably interpreted in terms of the EDL model. Note that the decrease in $I_{\mathrm{EMR}}$ occurs in the same manner for all frequencies encountered in the Fourier series (see Fig. 3). This result, though inconsistent with the relation $I_{\mathrm{EMR}} \propto \omega^{2}$ that follows from (3), is not surprising. For the relation $I_{\mathrm{EMR}} \propto \omega^{2}$ to hold for a test sample, it is necessary that the EMR signal generated by the sample be the total of the signals generated simultaneously by all EDLs present in the sample. As we dealt with an actual rock, EMR could hardly be generated simultaneously throughout the bulk of the sample. Thus, our observation that $I_{\mathrm{EMR}}$ decreased with increasing strength of the skarn samples at least does not contradict the conclusions that can be made based on the EDL model.

Sandstone. For the sandstone samples, we observed a pronounced trend for a decrease in EMR with increasing strength. For acoustically excited 
sandstone, the EMR generation mechanism is determined by the petrophysical and textural characteristics of the rock (Yavorovich et al. 1999). The petrophysical feature of the sandstone samples used in our study was that they contained the different size of the clast: medium size $(0.2-$ $0.6 \mathrm{~mm})$ and coarse size $(0.6-2 \mathrm{~mm})$ (Table 1). The textural feature of the test sandstone samples was their high porosity. The EMR signal resulting from acoustic excitation of a sandstone sample is an integral characteristic which is determined by the structure-textural features of the sample. Ultimate strength is higher for the sandstone with the coarse clasts and the small porosity (Table 2). The EDLs appear on the pore boundary which reduces the EDL number in the sample volume. The alternating pressure of the acoustic field acting on these EDLs results in the conversion of the acoustic pulse energy to the EMR energy. The EMR amplitude can be estimated using Eq. (3). This equation indicates that the EMR amplitude is inversely proportional to Young's modulus, which is proportional to the ultimate strength. Hence, the EMR amplitude should decrease with increasing ultimate strength. This is precisely what we observed for the sandstone samples (Table 2), and this trend can be explained in the context of the EDL model.

Magnetite ore. For the magnetite ore samples, as distinct from the skarn samples, no pronounced dependence of the EMR signal amplitude on $P_{\text {ult }}$ was observed. For instance, the increase in $P_{\text {ult }}$ by $20 \%$ for skarn samples S1 and S2 resulted in a threefold decrease in EMR signal amplitude, whereas the same increase in $P_{\text {ult }}$ for ore samples M1 and M2 gave the opposite effect: the EMR signal amplitude doubled (see Table 2). Also noteworthy is the greater EMR signal amplitude for samples M1 and M2 compared to the EMR signals obtained by sounding the skarn samples. We suppose that both of the above effects are due to the presence of crystalline quartz inclusions in magnetite ore. Previously, we carried out measurements for the EMR signal generated by a hammer excitation of magnetite ore samples free of crystalline quartz inclusions and magnetite ore samples containing $10 \%$ of crystalline quartz (Bespalko et al. 2005). It was observed that the increase in EMR signal amplitude measured for the magnetite ore containing quartz was tenfold that of the ore not containing quartz. Similar results were obtained by other researchers (Wan et al. 2008, Kobayashi et al. 2014). In particular, the authors of (Kobayashi et al. 2014), in their experiment on dynamic compression of rocks, observed that the EMR signal amplitude for gabbro with 2 vol.\% quartz content was one sixth of that for granite with 36 vol.\% quartz content. Crystalline quartz inclusions in magnetite ore are randomly distributed over the volume, and this may account for the great spread in EMR signal amplitude from sample to sample in our experiments. Probably, the peak of $90 \mathrm{kHz}$ frequency (Fig. 5b, d, and h) is related to the quartz inclusions in the magnetite ore sample. 


\section{CONCLUSION}

In our study presented in this paper, we focused on the secondary electromagnetic radiation that arises upon acoustic excitation of rocks with different content of the crystalline and amorphous inclusions. The study has shown that for these rocks, the amplitude of the EMR signal is related to the ultimate strength of the rock material. Namely, in the experiment on acoustic excitation of EMR signals in skarn and sandstone samples, it was observed that the EMR signal amplitude decreased with increasing ultimate strength of the samples. Supposedly, this effect can be explained supposing that the EMR was generated when the acoustic wave propagated through an electrical double layer. For the magnetite ore samples, the EMR signal amplitude showed no dependence on ultimate strength, and it was greater than that observed for the sounded skarn samples. The most probable reason for this difference is the presence of piezoelectric inclusions in the magnetite ore samples.

Acknowledgements. The authors are grateful to Tashtagol mine stuff V. Klimko and V. Shtirts for help in the sample analyses. This work was supported in part by the Ministry of Education and Science of the Russian Federation under the State Research Project and by RFBR Grant No. 14-08-00395.

References

Baddari, K., G.A. Sobolev, A.D. Frolov, and A.V. Ponomarev (1999), An integrated study of physical precursors of failure in relation to earthquake prediction, using large scale rock blocks, Ann. Geophys. 42, 5, 771-787, DOI: 10.4401/ ag-3758.

Baddari, K., A.D. Frolov, V. Tourtchine, F. Rahmoune, and S. Makdeche (2015), Effect of stress-strain conditions on physical precursors and failure stages development in rock samples, Acta Geophys. 63, 1, 62-102, DOI: 10.2478/ s11600-014-0206-9.

Bespal'ko, A.A., L.V. Yavorovich, and P.I. Fedotov (2005), Communication parameters of electromagnetic signals with electrical characteristics of rocks under acoustic and quasi-static exposures, Izv. Tomsk Polytech. Univ. 308, 7, 18-23 (in Russian).

Bespal'ko, A.A., L.V. Yavorovich, E.V. Viitman, P.I. Fedotov, and V.A. Shtirts (2010), Dynamoelectric energy transfers in a rock mass under explosion load in terms of the Tashtagol mine, J. Min. Sci. 46, 2, 136-142, DOI: 10.1007/s10913-010-0018-5. 
Borla, O., G. Lacidogna, E. Di Battista, G. Niccolini, and A. Carpinteri (2015) Electromagnetic Emission as Failure Precursor Phenomenon for Seismic Activity Monitoring Conference Proceedings of the Society for Experimental Mechanics Series 2015, Fracture, Fatigue, Failure, and Damage Evolution, Vol. 5, 221-229, DOI: 10.1007/978-3-319-06977-7_29.

Carpinteri, A., F. Cardone, and G. Lacidogna (2010), Energy emissions from failure phenomena: mechanical, electromagnetic nuclear, Exp. Mech. 50, 8, 1235 1243, DOI: 10.1007/s11340-009-9325-7.

Carpinteri, A., G. Lacidogna, O. Borla, and A. Manuello (2012), Electromagnetic and neutron emission from brittle rocks failure: Experimental evidence and geological implications, Sadhana, 37, 1, 59-78, DOI: 10.1007/s12046-0120066-4.

Carpinteri, A., G. Lacidogna, A. Manuello, and O. Borla (2013), Piezonuclear fission reactions from earthquakes and brittle rock failure: evidence of neutron emission and non-radioactive product elements, Exp. Mech. 53, 3, 345-365, DOI: $10.1007 / \mathrm{s} 11340-012-9629-\mathrm{x}$.

Cs'efalvay, G., and P. Sedl'ak (2012), Experimental study on feature selection using artificial AE. In: 30th European Conference on Acoustic Emission Testing \& 7th International Conference on Acoustic Emission University of Granada, 12-15 September.

Fidani, C. (2011), The Central Italy electromagnetic network and the 2009 L'Aquila earthquake: observed increases in anomalies, Geosciences 1, 1, 3-25, DOI: 10.3390/geosciences 1010003.

Frid, V., and K. Vozoff (2005), Electromagnetic radiation induced by mining rock failure, Int. J. Coal Geol. 64, 1-2, 57-65, DOI: 10.1016/j.coal.2005.03.005.

Frid, V., A. Rabinovitch, and D. Bahat (2003), Fracture induced electromagnetic radiation, J. Phys. D 36, 13, 1620-1628, DOI: 10.1088/0022-3727/36/13/330.

Gol'd, R.M., G.P. Markov, and P.G. Mogila (1975), Pulsed electromagnetic radiation of minerals and rock subjected to mechanical loading, Izv. Earth Phys. 7, 109-111.

Kani, K., T. Yamada, and M. Abe (1985), Hugoniot and electric resistivity measurement on amorphous Se. In: Proc. 4th Amer. Phys. Soc. Conf. on Shock Waves in Condensed Matter, Spokane, Washington, 22-25 July,. Plenum Press, N.Y., 477-482.

Khatiashvili, N.G., and M.E. Perel'man (1989), On the mechanism of seismoelectromagnetic phenomena and their possible role in the electromagnetic radiation during periods of earthquakes, foreshocks and aftershocks, Phys. Earth Planet Inter. 57, 1-2, 169-177, DOI: 10.1016/0031-9201(89)90226-4.

Kobayashi, H., K. Horikawa, K. Ogawa, and K. Watanabe (2014), Impact compressive and bending behavior of rocks accompanied by electromagnetic phenomena, Phil. Trans. Roy. Soc. A 372, 2023, 20130292, DOI: 10.1098/ rsta.2013.0292. 
Koktavy, P. (2009), Experimental study of electromagnetic emission signals generated by crack generation in composite materials, Meas. Sci. Technol. 20, 1, 015704-8, DOI: 10.1088/0957-0233/20/1/015704.

Koktavy, P., J. Pavelka, and J. Sikula (2004), Characterization of acoustic and electromagnetic emission sources, Meas. Sci. Technol. 15, 5, 973-977, DOI: 10.1088/0957-0233/15/5/028.

Lacidogna, G., A. Carpinteri, A. Manuello, G. Durin, A. Schiavi, G. Niccolini, and A. Agosto (2011), Acoustic and electromagnetic emissions as precursor phenomena in failure processes, Strain 47, Suppl. s2, 144-152, DOI: 10.1111/j.1475-1305.2010.00750.x.

Lacidogna, G., O. Borla, G. Niccolini, and A. Carpinteri (2013), Dynamic behavior of materials. In: Conference Proceedings of the Society for Experimental Mechanics Series, Vol. 1, 387-393.

Heiniö, M. (ed.) (1999), Excavation Engineering Handbook, Sandvik Tamrock Corp.

Mavko, G., T. Mukerji, and J. Dvorkin (2009), The Rock Physics Handbook, 2nd ed., Cambridge University Press, Cambridge.

Perel'man, M.E., and N.G. Khatiashvili (1983), Generation of electromagnetic radiation during oscillation of double electric layers and its manifestation at earthquakes, Dokl. Akad. Nauk SSSR 271, 80-83.

Ponomarev, A., G. Sobolev, and A. Koltsov (2002), Acoustic emission under electric excitation. In: ESC XXVIII General Assembly, Genova, Book of Abstracts, p. 238.

Scholz, C.H. (1968), Microfracturing and the inelastic deformation of rock in compression, J. Geophys. Res. 73, 4, 14-17, DOI: 10.1029/JB073i004p01417.

Sedlak, P., J. Sikula, T. Lokajicek, and Y. Mori (2008), Acoustic and electromagnetic emission as a tool for crack localization, Meas. Sci. Technol. 19, 4, 045701-7, DOI: 10.1088/0957-0233/19/4/045701.

Sobolev, G.A., A.V. Ponomarev, Yu.Ya. Maibuk, N.A. Zakrzhevskaya, V.I. Ponyatovskaya, D.G. Sobolev, A.A. Khromov, and Yu.V. Tsyvinskaya (2010), The dynamics of the acoustic emission with water initiation, Izv. Phys. Solid Earth 46, 2, 136-153, DOI: 10.1134/S1069351310020035.

Sobotka, J. (2004), The laboratory modelling of effect of electric and acoustic fields interaction in porous media saturated with water or hydrocarbons, Acta Geophys. Pol. 52, 3, 381-396.

Sobotka, J. (2009a), DC induce acoustic emission of saturated sand models of sedimentary rocks, Acta Geophys. 58, 3, 381-396, DOI: 10.2478/s11600-0090046-1.

Sobotka, J. (2009b), Longitudinal ultrasonic waves in DC electric field, Acta Geophys. 57, 2, 247-256, DOI: 10.2478/s11600-008-0072-4.

Wan, G.-X., X.-B. Li, and L. Hong (2008), Piezoelectric responses of brittle rock mass containing quartz to static stress and exploding stress wave respec- 
tively, J. Central South Univ. Technol. 15, 3, 344-349, DOI: 10.1007/ s11771-008-0065-0.

Warwick, J.W., C. Stoker, and T.R. Meyer (1982), Radio emission associated with rock fracture: possible application to the great Chilean earthquake of May 22, 1960, J. Geophys. Res. 87, B4, 2851-2859, DOI: 10.1029/ JB087iB04p02851.

Yavorovich, L.V., R.M. Gold, and V.V. Lasukov (1999), Investigating the amplitude of the electromagnetic signal on impact on the sample rocks with different porosity, Fiz.-Tekhn. Probl. Razrabotki Poleznykh Iskopaemykh 6, 33-39 (in Russian).

Received 3 April 2015

Received in revised form 8 February 2016

Accepted 5 April 2016 